\title{
ALIMENTOS TÓXICOS PARA CÃES E GATOS
}

Amália Turner Giannico ${ }^{1}$, Clara Alita corona Ponczek ${ }^{1}$, Aline Spina de Jesus ${ }^{2}$, Alessandra Melchert ${ }^{3}$, Priscylla Tatiana Chalfun Guimarães-Okamoto ${ }^{3}$

${ }^{1}$ Universidade Federal do Paraná - UFPR, Curitiba - PR. E-mail: amaliaturner@uol.com.br, claponczek@uol.com.br.

${ }^{2}$ Médica veterinária autônoma. ${ }^{3}$ Faculdade de Medicina Veterinária e Zootecnia - UNESP-Botucatu-SP. E-mail: alessandra@fmvz.unesp.br, tatiana@fmvz.unesp.br

\section{RESUMO}

Os animais de companhia estão cada vez mais próximos de seus donos e por este motivo acabam compartilhando de forma errônea a mesma alimentação. Várias substâncias são capazes de causar intoxicações ou envenenamentos nos cães e gatos, sendo que muitas delas estão presentes nos alimentos para consumo humano. Alguns alimentos que são comestíveis para os seres humanos e até mesmo para outras espécies animais podem representar riscos para cães e gatos devido às diferentes vias metabólicas, além das quantidades e concentrações consumidas. As intoxicações nos animais ocorrem de forma intencional ou acidental no domicílio do proprietário do animal, ocorrendo manifestação aguda dos sinais clínicos. O objetivo desta revisão foi apresentar os produtos alimentícios, para seres humanos, que não devem ser fornecidos para cães e gatos assim como sinais clínicos característicos de uma toxicose por estes alimentos, demonstrando também sua terapia geral. Esta revisão explora em detalhes os mecanismos de ação e os sinais clínicos decorrentes de toxicose causada por chocolate, xilitol, macadâmia, cebola, alho, uvas e passas, abacate, bebidas alcoólicas e leite.

Palavras-chave: intoxicação; pequenos animais; produtos alimentícios

\section{TOXIC FOOD FOR DOGS AND CATS}

\begin{abstract}
Pet animals are closer to their owners and for this reason erroneously share the same alimentation. Several substances can cause intoxication or poisoning in dogs and cats, being many of those also found in human food. Some foods that are edible for humans or even to other animal species can be risky for dogs and cats due to different metabolic pathways, and further damage can be caused depending on the amount or concentration consumed. Poisoning in animals occur intentionally or accidentally in the household of its owner, occurring acute onset of clinical signs. Thus, the objective of this review was to present the food products for humans that cannot be given to dogs and cats as well as clinical signs characteristic of a toxicosis, also demonstrating the therapy for the damage created by consumption of those substances. This review explores the mechanisms of action and clinical signs of toxicosis caused by chocolate, xylitol, macadamia nuts, onions, garlic, grapes and raisins, avocado, alcoholic beverages and milk.
\end{abstract}

Keywords: intoxication; small animals; food products. 


\section{INTRODUÇÃO}

Existem vários agentes que podem causar intoxicações nos cães e gatos. A gravidade das intoxicações nestes animais depende da disponibilidade desses agentes no meio ambiente, quantidade de agente ao qual o animal está exposto e sensibilidade individual para os seus efeitos (KOVALKOVIČOVÁ et al., 2009).

Cerca de $90 \%$ das intoxicações nos animais são acidentais e agudas, ocorrendo perto ou no domicílio do proprietário (GODBOLD et al., 1979; OGA, 2003; TODD; POWELL, 2007; KOVALKOVIČOVÁ et al., 2009; XIA et al., 2009). Existem alguns alimentos consumidos pelos seres humanos e até mesmo por outras espécies de animais que podem representar riscos para cães e gatos devido a diferente metabolização (KOVALKOVIČOVÁ et al., 2009). Os hábitos e apetite exigente dos gatos fazem com que a intoxicação alimentar nestes animais seja três vezes menos frequente do que nos cães (OGA, 2003; KOVALKOVIČOVÁ et al., 2009). Os alimentos tóxicos mais comuns para os gatos são cebola, alho, chocolate, uva, passas e alguns vegetais como tomate verde, batata verde ou cru. No caso dos cães, os produtos mais comuns que causam toxicoses são chocolate, café, doce que contém xilitol, nozes, macadâmia, cebola, alho, uva, passas, bebidas alcoólicas e leite (KOVALKOVIČOVÁ et al., 2009).

Esta revisão teve como objetivo apresentar os produtos alimentícios que não devem ser fornecidos para cães e gatos e os sinais clínicos característicos de uma toxicose por estes agentes e a terapia geral.

\section{REVISÃO DE LITERATURA}

\section{Chocolate, café e chá (teobromina)}

O chocolate é apreciado por muitas pessoas, inclusive pelos cães. Na constituição do chocolate existe grande quantidade de carboidratos, lipídios, aminas biogênicas, neuropeptídeos e metilxantinas (teobromina e cafeína, sendo a concentração de teobromina muito mais significativa do que a de cafeína). A manteiga de cacau é o ingrediente presente em maior quantidade no chocolate, e é ela que possui a teobromina (STIDWORTHY et al., 1997). A teobromina é uma substância com ação diurética, estimulante cardíaca e vasoconstritora, sendo extremamente nociva em excesso, podendo resultar em morte (BEASLEY, 1999; KOVALKOVIČOVÁ et al., 2009).

A teobromina é o componente tóxico mais importante do chocolate e provoca grande estimulação cerebral e intenso aumento no trabalho muscular cardíaco, ocasionando arritmias cardíacas importantes 
nos cães (STIDWORTHY et al., 1997, BEASLEY, 1999). A concentração dessa substância no chocolate pode ser de 3 a 10 vezes maior do que no café (BEASLEY, 1999). Ela também é encontrada no chá e bebidas à base de cola (KOVALKOVIČOVÁ et al., 2009). Quanto mais matéria lipídica possuir o chocolate, menor vai ser o teor de teobromina, como é o caso dos chocolates brancos, que não oferecem tanto risco para estes animais. Quanto mais escuro for o chocolate há mais teobromina, havendo uma maior possibilidade de ocorrer intoxicação (Tabela 1). Assim, os chocolates amargo e meio-amargo são os que oferecem maior risco, pois possuem um teor mais elevado desta substância (STIDWORTHY et al., 1997).

A quantidade de teobromina encontrada no chocolate é pequena o suficiente para que o chocolate possa ser consumido com segurança por pessoas em grandes quantidades, mas os cães metabolizam a teobromina mais lentamente, podendo facilmente se intoxicar (GORNIAK; SPINOSA, 2003).

As metilxantinas são bases altamente lipossolúveis (STIDWORTHY et al., 1997; GORNIAK; SPINOSA, 2003). Possuem a habilidade de atravessar as barreiras placentária e hematoencefálica e são absorvidas tanto no estomago quanto no intestino. No cérebro, competem com a adenosina, inibidor pré-sináptico, diminuindo sua ação inibitória e desta forma, causando excitação. A meia vida da teobromina no cão é de 17,5 horas, permanecendo no organismo por até seis dias (GORNIAK; SPINOSA, 2003). Este tempo de meia-vida é prolongado, pois sua excreção ocorre pela bile e não pela urina (STIDWORTHY et al., 1997; GORNIAK; SPINOSA, 2003).

Tabela 1. Quantidade de teobromina e cafeína em um grama de produto.

\begin{tabular}{lll}
\hline & Teobromina & Cafeína \\
\hline Chocolate branco & $0,009 \mathrm{mg}$ & $0,030 \mathrm{mg}$ \\
Chocolate ao leite & $2,046 \mathrm{mg}$ & $0,212 \mathrm{mg}$ \\
Chocolate meio-amargo & $4,586 \mathrm{mg}$ & $0,705 \mathrm{mg}$ \\
Chocolate amargo & $13,863 \mathrm{mg}$ & $1,658 \mathrm{mg}$ \\
Cacau em pó & $25,997 \mathrm{mg}$ & $2,469 \mathrm{mg}$ \\
\hline
\end{tabular}

Um cão de $10 \mathrm{~kg}$ pode ser seriamente (STIDWORTHY et al., 1997; KOVALKOVIČOVÁ intoxicado ao ingerir um quarto de um pacote de $250 \mathrm{~g}$ de cacau em pó et al., 2009). Quanto menor o cão, menor a quantidade necessária para intoxicar-se 
(GORNIAK; SPINOSA, 2003). Os efeitos dependem do peso do cão, da dosagem e do tipo de chocolate (STIDWORTHY et al., 1997; GORNIAK; SPINOSA, 2003).

As doses tóxicas de teobromina estão próximas a $100 \mathrm{mg} / \mathrm{kg}$ sendo fatais perto de $200 \mathrm{mg} / \mathrm{kg}$ (STIDWORTHY et al., 1997; KOVALKOVIČOVÁ et al., 2009). Porém, há relatos de sinais de intoxicação, como vômitos e diarréia, com ingestão de apenas $20 \mathrm{mg} / \mathrm{kg}$ (KOVALKOVIČOVÁ et al., 2009) e também de sinais de efeitos cardiotóxicos com ingestão de $50 \mathrm{mg} / \mathrm{kg}$ de teobromina (BEASLEY, 1999).

Há ainda, relatos de efeitos drásticos com a ingestão não só de chocolate em barra, mas também de chocolate em pó dissolvido em leite e oferecido aos cães. Os cães são os mais afetados por gostarem muito desta guloseima (KOVALKOVIČOVÁ et al., 2009), no entanto gatos também podem ser intoxicados ao ingerirem o chocolate (GORNIAK; SPINOSA, 2003).

\section{Mecanismo de ação}

Metilxantinas provocam um antagonismo competitivo dos receptores de adenosina (KOVALKOVIČOVÁ et al., 2009; GORNIAK; SPINOSA, 2003). A inibição destes receptores provoca estimulação do sistema nervoso central (SNC), a constrição de alguns vasos sanguíneos, diurese e taquicardia (BEASLEY, 1999; KOVALKOVIČOVÁ et al.,
2009). Metilxantinas também podem aumentar os níveis de cálcio intracelular, inibindo a fixação de cálcio pelo retículo sarcoplasmático dos músculos estriados (GORNIAK; SPINOSA, 2003). O efeito é o aumento da força e da contratilidade do músculo esquelético e cardíaco (GORNIAK; SPINOSA, 2003; KOVALKOVIČOVÁ et al., 2009). Essas substâncias também podem competir pelos receptores benzodiazepínicos no SNC e inibirem a fosfodiesterase, resultando no aumento dos níveis de adenosina monofosfato cíclico (AMP) (GORNIAK; SPINOSA, 2003). Também podem aumentar os níveis circulantes de epinefrina pela estimulação da zona medular das glândulas adrenais (STIDWORTHY et al., 1997; KOVALKOVIČOVÁ et al., 2009).

\section{Sinais clínicos}

Os sinais clínicos desta intoxicação geralmente ocorrem dentro de seis a 12 horas após a ingestão (STIDWORTHY et al., 1997). A teobromina, uma vez absorvida, pode permanecer ativa no corpo por 24 horas antes de ser eliminada (KOVALKOVIČOVÁ et al., 2009). A morte seguida da ingestão de doses fatais ocorre aproximadamente 24 horas mais tarde (STIDWORTHY et al., 1997; BEASLEY, 1999).

Podem ser observados náusea, êmese, diarréia, dispnéia, polidipsia, poliúria e polaquiúria (STIDWORTHY et al., 1997). 
Outros sinais clínicos e alterações avançadas que podem ser encontradas são desidratação, hiperatividade, arritmias cardíacas, hemorragias internas, hipertensão arterial sistêmica, taquipnéia, cianose, hipertermia, fraqueza, tremores, ataxia, convulsões, coma e morte (BEASLEY, 1999; GORNIAK; SPINOSA, 2003). O alto teor de gordura dos produtos de chocolate ainda pode provocar pancreatite em animais sensíveis (KOVALKOVIČOVÁ et al., 2009).

As intoxicações por café e chá estão relacionadas à cafeína, o que é bastante semelhante à teobromina encontrada no chocolate (STIDWORTHY et al., 1997; KOVALKOVIČOVÁ et al., 2009). Suas fontes incluem bebidas que contenham café, chás e até mesmo pílulas de cafeína (KOVALKOVIČOVÁ et al., 2009). Os sinais clínicos estão relacionados ao coração, pulmão, rim e SNC, como hiperatividade, êmese e dor abdominal no início, seguidos por estado ofegante, fraqueza, ataxia, aumento da frequência cardíaca, tremores musculares, convulsões, coma e morte (GORNIAK; SPINOSA, 2003; HOWLETT et al., 2005).

\section{Tratamento}

O tratamento da intoxicação por chocolate, café ou chá é de suporte. Deve-se eliminar as toxinas ainda não absorvidas, por meio da indução do vômito ou lavagem gástrica com uso de carvão ativado, caso a intoxicação tenha ocorrido há menos de 2 horas. Medidas como instituição de fluidoterapia, monitoração cardíaca e controle de convulsões são fundamentais. Uma vez que as metilxantinas podem ser reabsorvidas a partir da vesícula urinária, deve-se manter o animal sondado e a bexiga vazia. Devido à longa meia vida da teobromina, os sinais clínicos podem perdurar por 24 a 72 horas, devendo-se manter o animal internado até a recuperação total (ANDRADE, 2011).

\section{Xilitol}

O xilitol é um açúcar pentanol comumente utilizado como adoçante substituto do açúcar (sacarose) em muitos produtos, inclusive em produtos para diabéticos (KOVALKOVIČOVÁ et al., 2009). Também é utilizado como ingrediente em balas e gomas de mascar para a prevenção de cáries, especialmente nas crianças (HONKALA et al., 2006) e vem apresentando um aumento do seu uso na sua forma em pó para fabricação de pães. Além disso, o xilitol é encontrado em vários vegetais e frutas, como ameixas, morangos e framboesas (KITCHENS, 2005).

Os cães são sensíveis ao xilitol, e a ingestão de produtos que contenham essa substância pode levar à intoxicação 
(DUNAYER, 2004; TODD; POWELL, 2007; XIA et al., 2009).

O Centro de Controle de Envenenamento Animal da Sociedade Americana à Prevenção da Crueldade aos Animais (ASPCA) relatou o aumento da incidência da intoxicação por xilitol nos cães, mostrando que existiram, nos Estados Unidos, três casos em 2002, 20 em 2003, 82 em 2004, 193 em 2005 e 198 até julho de 2006 (DUNAYER; GWALTNEY-BRANT, 2006).

\section{Mecanismo de ação}

O xilitol é um metabólito intermediário do ciclo do ácido glucurônico que estimula a síntese e secreção da insulina, o que resulta nos cães em hiperinsulinemia (KUZUYA; KANAZAWA, 1969; DUNAYER, 2004). O mecanismo exato de como isso ocorre é desconhecido, mas demostrou-se em um estudo que o xilitol, por si só, estimula diretamente a secreção de insulina nas células $\beta$ da ilhota pancreática (KUZUYA; KANAZAWA, 1969).

A insulina estimula a atividade da enzima de membrana ATPase, que por sua vez, catalisa a transferência de íons potássio para o meio intracelular (AL-KHALILI et al., 2003), o que induz a hipocalemia. Além disso, a hiperinsulinemia levará a uma hipoglicemia, que tem pico entre 10 a 20 minutos após o pico de insulina sérica (KUZUYA; KANAZAWA, 1969; DUNAYER, 2004).
O xilitol é também metabolizado no fígado, entrando na via pentose-fosfato e produzindo um metabólito intermediário (ribose-5-fosfato), antes de ser transformado em glicose (GORNIAK; SPINOSA, 2003; DUNAYER; GWALTNEY-BRANT, 2006). Todo esse processo de metabolização requer adenosina trifosfato (ATP), e quando uma grande quantidade de xilitol é absorvida na circulação sanguínea, o ATP dos hepatócitos se esgota, conduzindo à necrose destes e ao consequente aumento sérico das enzimas alanina aminotransferase (ALT) e aspartato aminotransferase

(DUNAYER; GWALTNEY-BRANT, 2006). Estudo mais recente mostrou aumento da bilirrubina indireta (XIA et al., 2009), o que se deve à hemólise, pois sabe-se que o suprimento contínuo de glicose sanguínea é necessário para a integridade da membrana de leucócitos e eritrócitos (NAGY et al., 1998). Como o suprimento de glicose é reduzido em consequência da hipoglicemia, há ruptura da membrana dos eritrócitos, levando à liberação de hemoglobina, a qual é degradada em bilirrubina (NAGY et al., 1998). Além disso, há relato de estudo in vitro de que $\mathrm{o}$ xilitol pode promover aumento na concentração sérica de cálcio, pois afeta o tecido epitelial e aumenta a absorção de cálcio no intestino (MINEO et al., 2002). 


\section{Sinais clínicos}

A intoxicação por xilitol nos cães é caracterizada por depressão, vômito, ataxia e fraqueza, sendo a maioria desses sinais clínicos secundários à hipoglicemia, que normalmente é observada 30 a 60 minutos após a ingestão desta substância (DUNAYER, 2004). Há relato de que $0,15 \mathrm{~g} / \mathrm{kg}$ de xilitol foi o suficiente para causar hipoglicemia e levar, consequentemente, ao aparecimento dos sinais clínicos (DUNAYER; GWALTNEY-BRANT, 2006).

Segundo Xia et al. (2009), os animais que foram submetidos à administração de xilitol na dosagem de $1 \mathrm{~g} / \mathrm{kg}$ e $4 \mathrm{~g} / \mathrm{kg}$, apresentaram aumento na concentração sérica de insulina e diminuição da glicemia. ALT e AST também apresentaram aumento acentuado, proporcional à dose administrada. Não foram observadas alterações na gama-glutamil transpeptidase (GGT) e na bilirrubina conjugada, porém a bilirrubina total aumentou após uma a duas horas da administração. Isso sugere que o acometimento hepático não está relacionado à estase biliar, já o aumento da bilirrubina total deve-se ao fato da hemólise, como já visto anteriormente (NAGY et al., 1998). Também foi observada diminuição do potássio e fósforo inorgânico. O cálcio ficou mais elevado nos animais do grupo controle por três horas, porém não foi maior que os valores de referência, e o cloro e o sódio não sofreram alteração. Sendo assim, esse estudo mostrou que a intoxicação por xilitol é caracterizada por hiperinsulinemia, hipoglicemia, hipocalemia, hipofosfatemia, hiperbilirrubinemia, e aumento da concentração sérica de AST e ALT (XIA et al., 2009).

Todd e Powell (2007) relataram leve leucocitose neutrofílica e desidratação (comprovado pelo hematócrito elevado com hiperproteinemia) em um animal que ingerira 95 gramas $(3,7 \mathrm{~g} / \mathrm{kg})$ de xilitol. coagulograma revelou significante aumento no tempo de protrombina e da tromboplastina parcial ativada, e ainda diminuição no nível de fibrinogênio plasmático. A avaliação citológica do fígado mostrou marcantes mudanças degenerativas nas células hepáticas, alterações vacuolares não lipídicas e colestase. Todas essas alterações, juntamente ao quadro clínico do animal, levaram ao diagnóstico de intoxicação por xilitol causando insuficiência hepática aguda.

\section{Tratamento}

O tratamento da intoxicação por xilitol deve ser de suporte, ou seja, tratam-se as alterações ocasionadas pela hipoglicemia, como fluidoterapia e reposição de eletrólitos (DUNAYER; GWALTNEY-BRANT, 2006; XIA et al., 2009). Também é possível o uso de tratamento preventivo aos danos hepáticos, 
pelo uso de hepatoprotetores, como silimarina e ácido ursodesoxicólico e transfusão de plasma fresco congelado, principalmente quando há alterações na coagulação (TODD; POWELL, 2007).

$\mathrm{Na}$ maioria das vezes em que há suspeita de insuficiência hepática secundária à ingestão de xilitol o animal já não apresenta mais hipoglicemia no momento em que é examinado. Além disso, um tratamento precoce e agressivo aos danos hepáticos, mesmo quando o animal está assintomático, pode prevenir a mortalidade devido à insuficiência hepática aguda (TODD; POWELL, 2007).

\section{Macadâmia}

A macadâmia é a semente de um fruto obtido da árvore das espécies Macadamia integrifolia e Macadamia Tetraphylla (KOVALKOVIČOVÁ et al., 2009). Seu princípio tóxico ainda é desconhecido, mas sabe-se que muitas variedades de macadâmia contêm níveis tóxicos de glicosídeos cianogênicos, porém eles possuem sabor amargo e não são utilizados na alimentação (BOTHAA; PENRITHB, 2009).

\section{Mecanismo de ação}

O mecanismo de ação da intoxicação por macadâmia não é conhecido (HANSEN, 2002). Em seres humanos já foram relatadas reações anafiláticas (KNOTT et al., 2008), mas nenhuma outra sintomatologia semelhante a que ocorre nos cães, sendo que os sinais clínicos nos cães que ingeriram as nozes sugerem não se tratar de um processo alérgico (HANSEN et al., 2000).

Na maioria dos relatos de intoxicação, o consumo de cinco a 40 sementes de macadâmia foi suficiente para o aparecimento de sinais clínicos (BOTHAA; PENRITHB, 2009). A média de consumo para intoxicação nos cães foi de $11,7 \mathrm{~g} / \mathrm{kg}$ (variando de 2,2 a $62,4 \mathrm{~g} / \mathrm{kg}$ ), sendo que o tempo entre a ingestão e o aparecimento dos sinais clínicos foi menor que 12 horas em 79\% dos casos (HANSEN et al., 2000).

\section{Sinais clínicos}

Os sinais clínicos da intoxicação por macadâmia cursam inicialmente com fraqueza, depressão, vômito e hipertermia, evoluindo para ataxia e paresia, dor e edema dos membros pélvicos (HANSEN et al., 2000; HANSEN, 2002). Além disso, são observados tremores, dor abdominal, claudicação, rigidez das articulações e palidez das mucosas (HANSEN et al., 2000).

No estudo onde a intoxicação por macadâmia foi reproduzida em laboratório por meio da administração de $20 \mathrm{~g} / \mathrm{kg}$ desta semente, os cães apresentaram fraqueza intensa com a incapacidade de manter-se em estação por 12 horas após a administração. Exames laboratoriais realizados nestes 
animais mostraram apenas elevação sérica da lipase ativa (HANSEN et al., 2000). Na pesquisa realizada por Bothaa e Penrithb (2009) os animais apresentaram aumento transitório dos triglicerídeos.

O diagnóstico desta intoxicação deve ser baseado no histórico de ingestão, ou presença da semente no vômito ou fezes, além do aparecimento abrupto de fraqueza dos membros pélvicos, quando excluída possibilidade de trauma, neuropatia periférica, envolvimento do SNC ou dor muscular (HANSEN et al., 2000).

\section{Tratamento}

Não existe antídoto e deve ser instituído o tratamento suporte com fluidoterapia e outras medidas necessárias. O enema é indicado e a lavagem gástrica deve ser realizada até duas horas após a ingestão (ANDRADE, 2011).

O prognóstico é bom, já que na maioria dos casos o animal apresenta remissão dos sinais clínicos após 24 horas (HANSEN et al., 2000; HANSEN, 2002).

\section{Cebola e Alho}

A cebola (Allium cepa) é rica em componentes químicos que trazem benefícios para a saúde humana, como os flavonóides (SLIMESTAD et al., 2007). Além de seus usos culinários, a cebola tem propriedades medicinais que têm sido atribuídas desde os tempos antigos, o que fez com que fosse realizada análise química exata dos seus ingredientes ativos nos últimos anos (SLIMESTAD et al., 2007; KOVALKOVIČOVÁ et al., 2009). Estes benefícios para a saúde fornecidos por alguns compostos da cebola incluem propriedades anti-carcinogênica, antiplaquetária, atividade antitrombótica, antiasmático, antidiabético, efeitos fibrinolíticos e várias outras ações biológicas e antibióticas (SLIMESTAD et al., 2007). Por outro lado, a cebola contém os componentes tóxicos que podem danificar as células vermelhas do sangue e provocar anemia hemolítica acompanhada pela formação de corpúsculos de Heinz nos eritrócitos de mamíferos (DESNOYERS, 2000; TANG et al., 2008).

$\mathrm{O}$ alho (Allium sativum) é considerado menos tóxico e seguro para cães do que a cebola, quando usado com moderação. Os agentes farmacologicamente ativos do alho são a alicina e o ajoene, potentes relaxantes de músculos cardíacos e de músculo liso, agentes vasodilatadores e hipotensores (COPE, 2005).

O consumo de apenas $5 \mathrm{~g} / \mathrm{kg}$ de cebola pelos gatos, ou 15 a 30g/kg pelos cães, resultou em alterações hematológicas clinicamente importantes (COPE, 2005).

Mecanismo de ação 
O mecanismo de toxicidade da cebola tem sido estudado há várias décadas, mas estudos recentes tem mostrado que mais de uma toxina está envolvida (COPE, 2005; TANG et al., 2008). Os componentes tóxicos em todo o tipo de cebola, alho, alho-poró, cebolinha e outros vegetais da família Allium, são sulfóxidos e sulfetos alifáticos (COPE, 2005). É amplamente aceito que sulfureto de n-propil é a principal toxina que reduz a atividade da glucose-6-fosfato desidrogenase em células vermelhas do sangue, interferindo com a regeneração da glutationa necessária para evitar a desnaturação oxidativa da hemoglobina (THRALL, 2003). Hemoglobinas desnaturadas precipitam na superfície das células vermelhas do sangue (corpúsculos de Heinz) e desencadeiam a hemólise intra e extravascular (TANG et al., 2008).

Todas as formas de cebola podem ser tóxicas, incluindo: desidratada, cebola crua ou cozida, em pizzas, pratos chineses, ou seja, qualquer alimento que contenha cebola (COPE, 2005).

Dentre as espécies estudadas, o homem é a mais resistente (KOVALKOVIČOVÁ et al., 2009). Embora o cão pareça ser uma das espécies mais sensíveis, existem poucos relatos na literatura científica sobre 0 envenenamento acidental, associados à ingestão de cebola por estes animais (TANG et al., 2008; KOVALKOVIČOVÁ et al., 2009). Os gatos são mais suscetíveis do que os cães
(COPE, 2005). A alimentação diária de cebola poderia ter um efeito acumulativo devido à formação contínua de corpúsculos de Heinz em comparação a uma única exposição. Um grande espaço de tempo até a próxima ingestão permite que a medula óssea regenere as células vermelhas destruídas prematuramente (DESNOYERS, 2000; COPE, 2005).

\section{Sinais clínicos}

Os primeiros sinais são normalmente de gastroenterite, como vômito, diarréia, dor abdominal e perda de apetite, sendo que depressão e desidratação também são observadas (TANG et al., 2008). Os sinais clínicos associados à hemólise, como mucosas pálidas ou ictéricas, frequência respiratória aumentada, letargia, fraqueza e hemoglobinúria demoram alguns dias para ocorrer (THRALL, 2003; COPE, 2005). O exame hematológico pode revelar neutrofilia, linfopenia, anemia com corpúsculos de Heinz e metahemoglobinemia (DESNOYERS, 2000; THRALL, 2003; TANG et al., 2008).

Os efeitos letais são raros nos cães (COPE, 2005), mas deve-se evitar a exposição a qualquer tipo de $A$. cepa nestes animais e nas outras espécies de animais domésticos (KOVALKOVIČOVÁ et al., 2009).

Nos cães que receberam extrato de alho por via gástrica uma vez por dia durante sete dias, a contagem de eritrócitos, 
hematócrito e concentração de hemoglobina diminuíram. A formação de corpúsculos de Heinz também foi detectada, no entanto, nenhum cão desenvolveu anemia hemolítica (LEE et al., 2000).

\section{Tratamento}

Não existe antídoto específico, entretanto, deve ser realizado o tratamento suporte com o objetivo de diminuir os efeitos oxidativos do ingrediente ativo e evitar a lesão renal resultante da hemoglobinúria. Caso a ingestão tenha sido até duas horas, deve-se induzir a êmese. O carvão ativado pode ser utilizado com o objetivo de adsorver os compostos dissulfetos. Pode ser administrado precursores da glutationa, Nacetilcisteína ou SAMe, sendo que para hipoxemia grave ou anemia hemolítica pode ser realizada uma transfusão sanguínea ou uso de hemoglobina bovina polimerizada (Oxyglobin) (GFELLER, MESSONNIER, 2006).

\section{Uva e Passas}

A intoxicação de cães por uvas in natura ou passas emergiu como uma potencial preocupação nos últimos anos. Estudos apontam como causadora dessa toxicose uma toxina ainda desconhecida ou ainda um fator extrínseco associado (CAMPBELL, 2007). Atualmente há relatos que confirmam que a ingestão destas frutas pode causar lesão renal nos cães (GWALTNEY et al., 2001).

De uma forma geral, qualquer quantidade ingerida de uva ou passas deve ser considerada um problema. Valores estimados de uvas associados a lesões renais nos cães são próximos de $32 \mathrm{~g} / \mathrm{kg}$, já a quantidade de uvas passas associada à manifestação de sinais clínicos variam de 11 a 30g/kg (GWALTNEY et al., 2001). Apesar disso, há relatos de casos de óbito em cães quando a dose ingerida destes frutos foi de 5 a $10 \mathrm{~g} / \mathrm{kg}$ (CAMPBELL, 2007).

\section{Mecanismo de ação}

O princípio tóxico é ainda desconhecido (BOTHA, PENRITH, 2009) e seu mecanismo de ação ainda não foi elucidado. A aparente falta de uma relação entre dose e resposta clínica levou alguns autores a sugerir que isso possa refletir tanto a presença de um componente dos frutos presente em quantidades variáveis, quanto a existência de um composto que seja extrínseco aos frutos. Variações na resposta individual também podem ocorrer (KOVALKOVIČOVÁ et al., 2009). A investigação de casos individuais da intoxicação parece excluir a contaminação das uvas com pesticidas, metais pesados ou micotoxinas. Fatores como o teor excessivamente elevado de açúcar das uvas e passas, ocorrência de hipercalcemia, o possível envolvimento de substâncias 
vasoativas, assim como a suspeita de anafilaxia, foram postulados (BOTHA, PENRITH, 2009).

\section{Sinais clínicos}

Os sinais clínicos geralmente se manifestam dentro de 6 horas após ingestão, e no máximo dentro de 24 horas. Estes incluem diarréia, anorexia, dor abdominal, fraqueza, desidratação, tremores e letargia (GWALTNEY-BRANT et al., 2001; CAMPBELL, 2007). Polidipsia discreta associada à oligúria normalmente se desenvolve 72 horas após a ingestão do fruto, evoluindo para anúria (GWALTNEY-BRANT et al., 2001).

Na urinálise podem ser observados proteinúria, glicosúria e hematúria, mas raramente cristalúria. Pode-se observar também elevação de glicose, cálcio, fósforo e enzimas do fígado e pâncreas em alguns cães (GWALTNEY-BRANT et al., 2001). O prognóstico é reservado nos casos de lesão renal (GWALTNEY-BRANT et al., 2001; CAMPBELL, 2007).

\section{Tratamento}

Deve ser realizada a êmese se a intoxicação for recente, caso isso não seja possível pode ser instituída a lavagem gástrica. Tratamento suporte com fluidoterapia e avaliação do débido urinário, caso não tenha produção pode ser necessário a realização de diálise (GFELLER, MESSONNIER, 2006).

\section{Abacate}

Todas as partes da planta do abacate (Persea americana) como folhas, frutos e caroço são potencialmente tóxicos para os cães, gatos, camundongos, ratos, aves, coelhos, cavalos, bovinos e caprinos, entre outras espécies (BUORO et al.,1994).

Persin é uma toxina fungicida encontrada em todas as partes do abacateiro (BUORO et al.,1994; KOVALKOVIČOVÁ et al., 2009). Acredita-se que seja esta toxina a causadora das intoxicações por abacate nos animais quando ingerida em grande quantidade (BUORO et al.,1994). A dose letal não é conhecida e seu efeito é variável nas diferentes espécies (KOVALKOVIČOVÁ et al., 2009).

\section{Mecanismo de ação}

A persin pode causar necrose do epitélio da glândula mamária e do miocárdio. Entretanto, o mecanismo tóxico permanece desconhecido (HANDL, IBEN, 2010).

\section{Sinais Clínicos}

De um modo geral observa-se edema pulmonar e efusão pleural, causando dificuldade respiratória e morte por anóxia dentro de 24 horas após a ingestão . Também pode-se encontrar efusão pericárdica 
(BUORO et al.,1994). Além desta toxina, o alto teor de gordura presente no abacate também pode ser prejudicial, causando pancreatite (KOVALKOVIČOVÁ et al., 2009).

Animais intoxicados por abacate também podem apresentar irritação gastrointestinal, vômito e diarréia (BUORO et al.,1994).

\section{Tratamento}

Não há tratamento específico, deve ser instituído o tratamento sintomático e de suporte (Andrade, 2011).

\section{Álcool}

As bebidas alcoólicas contêm etanol, composto químico que causa depressão de SNC e sistema respiratório (RICHARDSON, 2006). O etanol é rapidamente absorvido sistemicamente, sendo importante procurar atendimento médico veterinário imediato. Intoxicações graves têm sido relatadas quando bebidas alcoólicas são fornecidas como uma brincadeira aos cães, que não toleram o álcool, mesmo em pequenas quantidades (VAN WUIJCKHUISE; CREMERS, 2003).

O etanol ou álcool etílico é o tipo de álcool mais comum. Está contido nas bebidas alcoólicas, é usado para limpeza doméstica e também como combustível para automóveis (VALENTINE, 1990). A intoxicação nos cães por etanol também foi relatada após a ingestão de frutas podres (VAN WUIJCKHUISE; CREMERS, 2003).

\section{Mecanismo de ação}

O mecanismo de ação sobre o SNC está relacionado, em parte, a alterações na permeabilidade da membrana celular dos axônios e sua provável ação facilitadora de sinapses pelo neurotransmissor ácido gamaaminobutírico (GABA) (VALENTINE, 1990; RICHARDSON, 2006).

O álcool aumenta a inibição sináptica mediada pelo GABA e pelo fluxo intracelular de íons cloreto (VALENTINE, 1990; RICHARDSON, 2006). Esse efeito do álcool e outras ações sedativas e motoras são inibidas pela bicuculina, um antagonista específico dos receptores GABAérgicos (VALENTINE, 1990). O álcool atua também sobre outros neurotransmissores além do GABA no sistema adrenérgico, fazendo com que aumentem a síntese e a liberação de noradrenalina; e no sistema opióide, diminuindo a ligação das encefalinas aos receptores enquanto aumenta os níveis de beta-endorfinas (VALENTINE, 1990).

\section{Sinais clínicos}

O álcool afeta rapidamente o SNC, causando nos seres humanos sedação, diminuição da ansiedade, ataxia, prejuízo da capacidade de julgamento e desinibição do comportamento (OGA, 2003). Os sinais de 
intoxicação incluem maior grau de sedação, depressão, letargia, fraqueza, andar cambaleante e hipotermia (VALENTINE, 1990; OGA, 2003). Nos animais, altas doses promovem ataxia, redução dos reflexos, alterações comportamentais, acidose metabólica, excitação ou depressão, diminuição da frequência respiratória e parada cardíaca podendo chegar à morte, assim como nas pessoas (OGA, 2003, VAN WUIJCKHUISE; CREMERS, 2003). No entanto, os cães possuem uma taxa metabólica elevada, sendo mais suscetíveis à intoxicação (RICHARDSON, 2006).

\section{Tratamento}

Devido a depressão do sistema nervoso central a êmese não é indicada pois aumenta o risco de aspiração. O uso de carvão ativado não tem sucesso pois o cão não adsorve bem o álcool e a lavagem gástrica, apesar de pouco eficaz, é indicada em caso de ingestão de grandes quantidades (GFELLER, MESSONNIER, 2006).

\section{Leite}

Estudos realizados mostram que tanto os cães como os gatos adultos não produzem lactase, enzima degradadora da lactose, sendo esta presente apenas nos filhotes (GASCHEN; MERCHANT, 2011).

Sinais clínicos
Portanto, de uma forma geral, o quadro clínico apresentado é o de diarréia, semelhante ao que ocorre com seres humanos que possuem intolerância a lactose (GUILFORD, 1994; GASCHEN; MERCHANT, 2011). Deste modo, a retirada da administração do leite ou ingestão de derivados é essencial para que não ocorra o quadro de diarréia e desidratação secundariamente. Nos casos mais graves de intoxicação por leite a realização de fluidoterapia como suporte deverá ser cogitada (GUILFORD, 1994).

\section{Tratamento}

O tratamento é a terapia suporte e retirada da fonte de lactose (Andrade, 2011).

\section{Terapêutica}

O tratamento para as intoxicações por alimentos inclui suporte agressivo com fluidoterapia, devendo-se realizar medidas de descontaminação, como indução do vômito ou realização de lavagem gástrica até duas horas após a ingestão da substância e a administração de carvão ativado, para reduzir a absorção do agente (VIANA, 2007; KOVALKOVIČOVÁ et al., 2009). Ao mesmo tempo, deve-se visar à estabilização dos parâmetros do paciente. A terapia de suporte objetiva manter a homeostase e facilitar a eliminação do agente tóxico, com fluidoterapia e diurese forçada com 
furosemida; tratamento taquiarritmias se necessário, tratamento de desequilíbrios eletrolíticos, principalmente a hipocalemia que pode ocorrer a partir de vômitos prolongados (RICHARDSON, 2006; VIANA, 2007). Neste caso também é recomendada a administração de antieméticos, como metoclopramida ou Cerenia ${ }^{\circledR}$ (maropitnat) (VIANA, 2007). Evitar administração concomitante de corticosteróides perante intoxicações por café ou chocolate, pois estes fármacos podem interferir na excreção de metilxantinas (KOVALKOVIČOVÁ et al., 2009). É recomendado o uso de antioxidantes (vitamina E e ácido ascórbico) como prevenção da formação de corpúsculos de Heinz e dano oxidativo nos gatos, quanto ocorrer ingestão de cebola ou alho. Em animais gravemente intoxicados por estes vegetais a transfusões sanguíneas tem sido empregadas com sucesso (HILL et al., 2001). Em animais alcoolizados, além da terapia de suporte também é recomendada a administração de soro glicosado, para reduzir os efeitos de narcose (VAN WUIJCKHUISE; CREMERS, 2003).

\section{CONSIDERAÇÕES}

Atualmente estão amplamente disponíveis no mercado, em diversas faixas de preço, rações comerciais para cães e gatos, que possuem todos os nutrientes para atuar como um alimento completo, não havendo a necessidade de fornecer aos animais quaisquer elementos destinados à alimentação humana.

\section{CONCLUSÃO}

O aumento considerável de intoxicações alimentares nos animais de companhia se deve ao fato destes estarem mais próximos a seus donos, e desta maneira, compartilharem a mesma alimentação. O mercado oferece ao seu público uma gama de produtos específicos, que muitas vezes imitam os alimentos de consumo humano e são rigorosamente balanceados, justamente para que não ocorra nenhum mal à saúde animal.

\section{REFERÊNCIAS}

AL-KHALILI, L.; KROOK, A.; CHIBALIN, A. V. Phosphorylation of the $\mathrm{Na}+\mathrm{K}+$-ATPase in skeletal muscle: potential mechanism for changes in pump cell-surface abundance and activity. Annals of the New York Academy of Sciences, v. 986, n. 1, p. 449-452, 2003. http://dx.doi.org/10.1111/j.17496632.2003.tb07228.x

ANDRADE, S.F. Intoxicação alimentar em pequenos animais. In: NOGUEIRA, R.M.B; ANDRADE, S.F. Manual de Toxicologia Veterinária. São Paulo: Editora Roca, 2011. P. 230-241.

BEASLEY, V. Toxicants associated with stimulation or seizures. In: Veterinary toxicology. International Veterinary information service, 1999.

BOTHAA, C.J.; PENRITHB, M. L. Potential plant poisonings in dogs and cats in southern Africa. Journal of the South African 
Veterinary Association, v. 80, n. 2, p. 63-74, 2009.

http://dx.doi.org/10.4102/jsava.v80i2.173

BUORO, I. B.; NYAMWANGE, S. B.; CHAI, D.; MUNYUA, S. M. Putative avocado toxicity in two dogs. Onderstepoort. Onderstepoort Journal of Veterinary Research, v. 61, n. 1, p. 107-109, 1994.

CAMPBELL, A. Grapes, raisins and sultanas, and other foods toxic to dogs. UK Vet, v. 12, n. 1, p. 1-3, 2007.

COPE, R. B. Allium species poisoning in dogs and cats. Journal of Veterinary Medicine, $\mathrm{v}$. 100, n. 8, p. 562-566, 2005.

DESNOYERS, M. Anemias associated with Heinz bodies. In: FEDMAN, B. F.; ZINKL, J. G.; JAIN, N. C. Schalm's Veterinary Hematology. 5. ed. Baltimore: Lippincott Williams \& Wilkins; 2000. p.178-80.

DUNAYER, E. K. Hypoglycemia following canine ingestion of xylitol-containing gum. Veterinary \& Human Toxicology, v. 46, n. 2, p. 87-88, 2004.

DUNAYER, E. K.; GWALTNEY-BRANT, S. M. Acute hepatic failure and coagulopathy associated with xylitol ingestion in eight dogs. American Veterinary Medical Association, v. 229, n. 7, p. 1113-1117, 2006. http://dx.doi.org/10.2460/javma.229.7.1113

GASCHEN, F. P.; MERCHANT, S. R. Adverse food reactions in dogs and cats. Veterinary Clinics of North America: Small Animal Practice, v. 41, n. 2, p. 361-379, 2011. http://dx.doi.org/10.1016/j.cvsm.2011.02.005

GFELLER, R. W.; MESSONNIER, S. P. Toxicologia e envenenamentos em pequenos animais. 2.ed. Roca: São Paulo, 2006. 376p.

GODBOLD, J. C. JR.; HAWKINS, B. J.; WOODWARD, M. G. Acute oral marijuana poisoning in the dog. Journal of the
American Veterinary Medical Association, v. 175, n. 10, p. 1101-1102, 1979.

GORNIAK, S. L.; SPINOSA, H. S. Farmacologia Veterinária: Considerações sobre farmacocinética que contribuem para explicar as diferenças de respostas observadas entre espécies animais. Revista CFMV, v. 9, n. 30, 2003.

GUILFORD, W. G. Nutritional management of gastrointestinal tract diseases of dogs and cats. Journal of Nutrition, v. 124, n. 12, p. 2663-2669, 1994.

GWALTNEY-BRANT, S.; HOLDING, J. K.; DONALDSON, C. W.; EUBIG, P. A.; KHAN, S. A. Renal failure associated with ingestion of grapes or raisins in dogs. Journal of the American Veterinary Medical Association, v. 218, n. 10, p. 1555-1556, 2001.

HANDL, S.; IBEN, C. Foodstuffs toxic to small animals - a review. The European Journal of Companion Animal Practice, v. 20, n.1, p. 3644, 2010.

HANSEN, S. R. Macadamia nut toxicosis in dogs. Journal of Veterinary Medicine, v. 97, n. 4, p. 274-276, 2002.

HANSEN, S. R.; BUCK, W. B.; MEERDINK, G.; KHAN, S. A. Weakness, tremors, and depression associated with macadamia nuts in dogs. Veterinary \& Human Toxicology, v. 42, n. 1, p. 18-21, 2000.

HILL, A. S.; O'NEILL, S.; ROGERS, Q. R.; CHRISTOPHER, M. M. Antioxidant prevention of Heinz body formation and oxidative injury in cats. American Journal of Veterinary Research, v. 62, n. 3, p. 370-374, 2001. http://dx.doi.org/10.2460/ajvr.2001.62.370

HONKALA, E.; HONKALA, S.; SHYAMA, M.; ALMUTAWA, S. A. Field trial on caries prevention with xylitol candies among disabled school students. Caries Research, v. 40, n. 6, p. 508-513, 2006. http://dx.doi.org/10.1159/000095650 
p.

HOWLETT, R.; KELLEY, K.; GRASSI, B.; GLADDEN, B.; HOGAN, B. Caffeine administration results in greater tension development in previously fatigued canine muscle in situ. Experimental Physiology, v. 90, n. 6, p. 873-879, 2005. http://dx.doi.org/10.1113/expphysiol.2005.0315 $\underline{59}$

KITCHENS, D. H. Xylitol in the prevention of oral diseases. Special Care in Dentistry, v. 25, n. $3, \quad$ p. 140-144, 2005. http://dx.doi.org/10.1111/j.17544505.2005.tb01424.x

KNOTT, E.; GÜRER, C. K.; ELLWANGER, J.; RING, J.; DARSOW, U. Macadamia nut allergy. Journal of the European Academy of Dermatology and Venereology, v. 22, n. 11, p. 1394-1395, 2008. http://dx.doi.org/10.1111/j.1468$\underline{3083.2008 .02657 . x}$

KOVALKOVIČOVÁ, N.; ŠUTIAKOVÁ, I.; PISTL, J.; ŠUTIAK, V. Some food toxic for pets. Interdisciplinary Toxicology, v. 2, n. 3. p. 169-176, 2009. http://dx.doi.org/10.2478/v10102-009-0012-4

KUZUYA, T.; KANAZAWA, Y. Studies on the mechanism of xylitol-induced insulin secretion in dogs. Effect of its infusion into the pancreatic artery, and the inhibition by epinephrine and diazoxide of xylitol-induced hyperinsulinaemia. Journal of Diabetology, $v$. 5, n. 4, p. 248-257, 1969. http://dx.doi.org/10.1007/BF01212093

LEE, K. W.; YAMATO, O.; TAJIMA, M.; KURAOKA, M.; OMAE, S.; MAEDE, Y. Hematologic changes associated with the appearance of eccentrocytes after intragastric administration of garlic extract to dogs. American Journal of Veterinary Research, v. 61, n. 11, p. 1446-1450, 2000. http://dx.doi.org/10.2460/ajvr.2000.61.1446

MINEO, H.: HARA, H.; TOMITA, F. Sugar alcohols enhance calcium transport from rat small and large intestine epithelium in vitro. Digestive Diseases and Sciences, v. 47, n. 6,
http://dx.doi.org/10.1023/A:1015378732294

NAGY, S.; PAÁL, M.; KÕSZEGI, T.; LUDÁNY, A.; KELLERMAYER, M. ATP and integrity of human red blood cells. Physiological chemistry and physics and medical NMR, $\mathrm{V}$. 30, n. 2, p. 141-148, 1998.

OGA, S. Fundamentos de toxicologia. 2. ed. São Paulo: Editora Atheneu, 2003.

RICHARDSON, J. A. Ethanol. In: PETERSON, M.; TALCOTT, P. Small Animal Toxicology. St. Luis: Elsevier Saunders; 2006. p.698-701.

SLIMESTAD, R.; FOSSEN, T.; VÅGEN, I. M. Onions: a source of unique dietary flavonoids. Journal of Agricultural and Food Chemistry, v. 55, n. 25, p. 10067-10080, 2007. http://dx.doi.org/10.1021/jf0712503

STIDWORTHY, M. F.; BLEAKLEY, J. S.; CHEESEMAN, M. T.; KELLY, D. F. Chocolate poisoning in dogs. Veterinary Record, v. 141, n. 1, p. 28, 1997.

TANG, X.; XIA, Z.; YU, J. An experimental study of hemolysis induced by onion (Allium cepa) poisoning in dogs. Journal of Veterinary Pharmacology and Therapeutics, v. 31, n. 2 , p. $143-149,2008$. http://dx.doi.org/10.1111/j.1365-

2885.2007.00930.x

THRALL, M. A. Hematologia Veterinária. In: TILLEY, L. P.; SMITH, J. R. Consulta Veterinária em cinco minutos: Espécie canina e felina. 2. ed. São Paulo: Editora Manole, 2003. p.618.

TODD, J. M.; POWELL, L. L. Xylitol intoxication associated with fulminant hepatic failure in a dog. Journal of Veterinary Emergency and Critical Care, v. 17, n. 3, p. 286-289, 2007. http://dx.doi.org/10.1111/j.14764431.2007.00243.x

VALENTINE, W. M. Toxicology of selected pesticides, drugs, and chemicals. Short-chain alcohols. Veterinary Clinics of North 
America: Small Animal Practice, v. 20, n. 2, p. 515-523, 1990.

VAN WUIJCKHUISE, L.; CREMERS, G. G. Alcohol poisoning in dogs. Tijdschr Diergeneeskd, v. 128, n. 9, p. 284-285, 2003.

VIANA, F. A. B. Guia Terapêutico Veterinário. 2. ed. Lagoa Santa, MG: Editora Cem; 2007.

$X I A$, Z.; HE, Y.; YU, J. Experimental acute toxicity of xylitol in dogs. Journal of Veterinary Pharmacology and Therapeutics, v. $32, \quad$ n. 5 , p. 465-469, 2009. http://dx.doi.org/10.1111/j.1365-

2885.2009.01065.x 